\title{
INJECTIVE OBJECTS IN THE CATEGORY OF $p$-RINGS
}

\author{
DAVID C. HAINES
}

\begin{abstract}
A $p$-ring (or generalized Boolean ring) $P$ is a ring of fixed prime characteristic $p$ in which $a^{D}=a$ for all $a$ in $P$. In this paper $P$ is partially ordered by a relation which is a generalization of the usual Boolean order. A subset $S$ of $P$ is then called quasiorthogonal if $a b(a-b)=0$ for all $a, b$ in $S$. It is shown that $P$ is injective in the category of $p$-rings if and only if every quasiorthogonal subset has a supremum under this partial order.
\end{abstract}

Sikorski [4] has shown that in the category $\mathscr{B}$ of Boolean rings the injective objects are the complete Boolean rings. The purpose of this paper is to present a generalization of this result to the category $\mathscr{P}$ of $p$-rings, where $\mathscr{P}$ is understood to be the category with objects rings $P$ of fixed prime characteristic $p$ in which $a^{p}=a$ for all $a \in P$ and with morphisms the usual ring homomorphisms.

If $P$ is a $p$-ring, then the set $B(P)$ of idempotents of $P$ is a Boolean ring under the multiplication of $P$ and the new addition defined by $a \oplus b=a+$ $b-2 a b$. Batbedat [2] has used $B$ to establish an isomorphism between $\mathscr{P}$ and $\mathscr{B}$. (See also Stringall [6].) Hence, the injective objects in $\mathscr{P}$ are simply those $P$ for which $B(P)$ is a complete Boolean ring. In this paper the injective objects in $\mathscr{P}$ will be characterized by a kind of completeness of a particular partial order that is an extension of the usual partial order on the Boolean ring of idempotents.

Definition 1. For $a, b \in P, a \leqq b$ if and only if $a^{p-1} b=a$.

It is easily shown that $\leqq$ is a partial order on $P$ (see, e.g., Abian [1]), but in general $(P, \leqq)$ is not a lattice. It is, however, a lower semilattice with meet defined by $a \wedge b=a-a(a-b)^{p-1}$. A simple calculation shows that $a(b \wedge c)=a b \wedge a c$ and $a \wedge 0=0$ for $a, b, c \in P$.

Foster [3] has shown that every element $a$ of $P$ is uniquely expressible as a sum of elements $\left\{e_{j}(a): j \in Z_{p}\right\}$ of $B(P)$. In particular,

$$
a=\sum_{j=1}^{p-1} j e_{j}(a)
$$

Presented to the Society, January 26, 1973 under the title Injectivity in the category of p-rings; received by the editors February 27, 1973.

AMS (MOS) subject classifications (1970). Primary 06A70; Secondary 06A40, $06 \mathrm{~A} 23$.

Key words and phrases. Category of $p$-rings, p-rings, injectivity, Boolean rings. 
where

$$
e_{j}(a)=\left(a-a\left(a-j a^{p-1}\right)^{p-1}\right)^{p-1}
$$

and

$$
e_{j}(a) e_{k}(a)=0 \quad \text { if } j \not \equiv k(\bmod p) .
$$

The following lemma provides an alternative representation of $a$ as the sum of meets of elements of $P$.

LEMMA 1. If $a \in P$ and $j \in Z_{p}$, then $j e_{j}(a)=a \wedge j a^{p-1}$.

Proof. First observe that if $b \leqq a$, then for any integer $n \geqq 1$, $a^{n}=((a-b)+b)^{n}=(a-b)^{n}+A+b^{n}$, where $A$ has a factor of $b(a-b)=$ $b\left(a-b^{p-1} a\right)=0$. Hence $a^{n}-b^{n}=(a-b)^{n}$. Letting $b=a\left(a-j a^{p-1}\right)^{p-1}$ and $n=p-1$, it follows that

$$
j e_{j}(a)=j\left(a^{p-1}-a^{p-1}\left(a-j a^{p-1}\right)^{p-1}\right)=a \wedge j a^{p-1} .
$$

In general, not every pair of elements of $P$ has a join. It is easily shown, however, that $a$ and $b$ have a join in $P$ if $a b(a-b)=0$. In particular, $a \vee b=a+b-a^{p-1} b$. (Note that $a b(a-b)=0$ is equivalent to $a^{p-1} b=a b^{p-1}$.) More generally, if the set $Q$ has an upper bound, then $a b(a-b)=0$ for all $a, b \in Q$.

Definition 2. Elements $a, b$ in $P$ are said to be quasi-orthogonal if $a b(a-b)=0$. A subset $Q$ of $P$ is called quasi-orthogonal if its elements are pairwise quasi-orthogonal. $P$ is then called quasi-orthogonally complete if every quasi-orthogonal subset has a supremum in $P$.

We now show that quasi-orthogonal elements have an important property in terms of the idempotents given in (2).

LEMMA 2. If $a b(a-b)=0$ then $e_{j}(a) e_{k}(b)=0$ for $j \not \equiv k(\bmod p)$.

Proof. We may assume $j, k \neq 0(\bmod p)$. Then

$$
\begin{aligned}
j e_{j}(a) k e_{k}(b) & =\left(a \wedge j a^{p-1}\right)\left(b \wedge k b^{p-1}\right) \\
& =a b \wedge j a^{p-1} b \wedge k a^{p-1} b \wedge j k a^{p-1} b^{p-1} .
\end{aligned}
$$

But for any $x$ in $P, k x \wedge j x=k x-k x(k x-j x)^{p-1}=k x-k x(k-j)^{p-1} x^{p-1}=0$, and letting $x=a b^{p-1}$, we obtain $j e_{j}(a) k e_{k}(b)=0$ and hence $e_{j}(a) e_{k}(b)=0$.

The next lemma makes use of the well-known fact (see, e.g., Sikorski [5, p. 60]) that if $S$ is a subset of a Boolean ring $R$ with supremum in $R$ and if $m \in R$, then

$$
m(\sup S)=\sup (m S)
$$


LEMMA 3. If $Q$ is a quasi-orthogonal subset of $P$ such that $\sup \left\{e_{j}(a)\right.$ : $a \in Q\}$ is in $P$, then

$$
\sup \left\{b^{p-1} e_{j}(a): a \in Q\right\}=e_{j}(b) \text { for all } b \in Q, j \in Z_{p} .
$$

Proof. Note first that

$$
\begin{aligned}
b^{p-1} e_{j}(a) & =\left(b e_{j}(a)\right)^{p-1}=\left(\sum_{j=1}^{p-1} k e_{k}(b) e_{j}(a)\right)^{p-1} \\
& =\left(j e_{j}(b) e_{j}(a)\right)^{p-1}=e_{j}(b) e_{j}(a),
\end{aligned}
$$

using (1) and Lemma 2. Hence

$$
\begin{aligned}
e_{j}(b) & =e_{j}(b) \sup \left\{e_{j}(a): a \in Q\right\} \\
& =\sup \left\{e_{j}(b) e_{j}(a): a \in Q\right\}=\sup \left\{b^{p-1} e_{j}(a): a \in Q\right\} .
\end{aligned}
$$

We are now ready to prove an important theorem relating the completeness of $B(P)$ to the quasi-orthogonal completeness of $P$. For brevity, sums are assumed to be taken over $j, k=1, \cdots, p-1$.

THEOREM. If $P$ is a p-ring and $B(P)$ the Boolean ring of idempotents of $P$, then $P$ is quasi-orthogonally complete if and only if $B(P)$ is complete.

Proof. We first observe that since any subset $S$ of $B(P)$ is quasiorthogonal, if $P$ is quasi-orthogonally complete, then sup $S \in P$. However $(\sup S)^{2}=\sup S$ from (4), so that sup $S \in B(P)$ and $B(P)$ is complete.

Conversely, let $Q$ be a quasi-orthogonal subset of $P$ and $s=\sum j \sup \left\{e_{j}(a)\right.$ : $a \in Q\}$. Because $e_{j}(a) \in B(P)$ for $a \in P$ and $j \in Z_{p}$, the various suprema are in $P$ and hence $s$ is in $P$.

Now choose $s \in Q$. Then $x^{p-1} \in B(P)$ and so

$$
\begin{aligned}
x^{p-1} s & =\sum j x^{p-1} \sup \left\{e_{j}(a): a \in Q\right\} \\
& =\sum j \sup \left\{x^{p-1} e_{j}(a): a \in Q\right\}=\sum j e_{j}(x)=x,
\end{aligned}
$$

using (4), Lemma 3, and (1). Thus $x \leqq s$ and $x$ is an upper bound of $Q$.

On the other hand, assume $u$ is any upper bound of $Q$. It follows from (4) and Lemma 2 that

$$
\sup \left\{e_{i}(a): a \in Q\right\} \cdot \sup \left\{e_{j}(a): a \in Q\right\}=0 \quad \text { if } i \not \equiv j(\bmod p) .
$$

Hence,

$$
s^{p-1} u=\sum j^{p-1} u\left(\sup \left\{e_{j}(a): a \in Q\right\}\right)=\sum\left(\sum k \sup \left\{e_{k}(u) e_{j}(a): a \in Q\right\}\right)
$$

from (1) and (4). But if $a \leqq u$, then $a^{p-1} u=a u^{p-1}$ and so from Lemma 2, $e_{k}(u) e_{j}(a)=0$ if $k \neq \equiv j(\bmod p)$. Also, a straightforward calculation shows that $e_{j}(u) e_{j}(a)=e_{j}(a)$, so $s^{p-1} u=\sum j \sup \left\{e_{j}(a): a \in Q\right\}=s$. Consequently, $s \leqq u$ and so $s$ is the supremum of $Q$ in $P$. 
In light of the above theorem and the remarks in the introductory paragraphs, we obtain the following corollary.

COROLlaRY. A p-ring is an injective object in the category of p-rings if and only if it is quasi-orthogonally complete.

\section{REFERENCES}

1. Alexander Abian, Direct product decomposition of commutative semi-simple rings, Proc. Amer. Math. Soc. 24 (1970), 502-507. MR 41 \#3461.

2. A. Batbedat, $p$-anneaux, Secrétariat des Math. de la Faculté des Sciences de Montpellier, 1968-1969, No. 34, Univ. de Montpellier, Montpellier, 1968. MR 39 \#5551.

3. A. L. Foster, p-rings and their Boolean vector representation, Acta Math. 84 (1951), 231-261. MR 12, 584.

4. R. Sikorski, A theorem on extension of homomorphisms, Ann. Soc. Polon. Math. 21 (1948), 332-335. MR 11, 76.

5. - Boolean algebras, 3rd ed., Ergebnisse der Math. und ihrer Grenzgebiete, Heft 25, Springer-Verlag, New York, 1969. MR 39 \#4053.

6. R. W. Stringall, The categories of p-rings are equivalent, Proc. Amer. Math. Soc. 29 (1971), 229-235. MR 43 \#1901.

Department of Mathematics, Bates College, Lewiston, Maine 04240 